\title{
Análise da Metodologia SOESGOPE aplicada a um Quadrotor para Estimação Ótima de Parâmetros
}

\author{
Pablo Henrique Gonçalves, Augusto Santiago Cerqueira, \\ Exuperry Barros Costa \\ Faculdade de Engenharia Elétrica \\ Universidade Federal de Juiz de Fora \\ Juiz de Fora, MG, Brasil, (e-mail: pablo.autufop@gmail.com, \\ augusto.santiago@ufjf.edu.br, exuperry.costa@ufjf.edu.br).
}

\begin{abstract}
:
In order for UAVs to be able to operate satisfactorily, robust control methods must be employed, and these are dependent on a faithful mathematical model of the system. This work presents an analysis of the SOESGOPE methodology for estimation of the parameters of a quadrotor and excitation signal generation for the experiment. The methodology consists of two layers of optimization: one internal, responsible for the optimal estimation of the parameters, and based on safety barrier interior point algorithm; and the outer, responsible for obtaining a (sub)optimal signal of excitation, with respect to metrics such as duration, space, excitation persistence and state space constraints. The outer layer is based on an algorithm similar to the Particle Swarm. In order to receive an excitation signal, a simple PID controller is proposed for the aircraft, tuned via the Twiddle method. Three simulation scenarios are analyzed, regarding the importance of knowledge of initial parameters, the uncertainty of the plant parameters, and finally a sensitivity analysis of the methodology with respect to its weighting gains. The SOESGOPE methodology presents positive results for application in VAANTS, being effective in the estimation of the parameters in all the scenarios.
\end{abstract}

\section{Resumo:}

Para que os VAANTs possam operar satisfatoriamente, robustos métodos de controle devem ser empregados, e estes são dependentes de um modelo matemático fiel do sistema. Este trabalho apresenta uma análise da metodologia SOESGOPE para estimação dos parâmetros de um quadrotor e geração de sinal de excitação para o experimento. A metodologia consiste de duas camadas de otimização: uma interna, responsável pela estimação ótima dos parâmetros, e baseada em algoritmo de pontos interiores com barreira de segurança; e a externa, responsável pela obtenção de um sinal (sub)ótimo de excitação, com relação a métricas como duração, espaço, persistência de excitação e restrições no espaço de estados. A camada externa é baseada em um algoritmo similar ao Particle Swarm. Para que possa receber um sinal de excitação, é proposto um controlador PID simples para a aeronave, sintonizado através do método twiddle. São analisados 3 cenários em simulação, relativos a importância de conhecimento de parâmetros iniciais, à incerteza dos parâmetros da planta, e por fim uma análise de sensibilidade da metodologia com relação a seus ganhos de ponderação. A metodologia SOESGOPE apresenta resultados positivos para aplicação em VAANTS, sendo eficaz na estimação dos parâmetros em todos os cenários.

Keywords: Quadrotor; Systems identification; Optimal Parameter Estimation; Optimal Input Design; Twiddle Method

Palavras-chaves: Quadrotor; Identificação de sistemas; Estimação Ótima de Parâmetros; Projeto de Entradas Ótimas; Método Twiddle 


\section{INTRODUÇÃO}

Os VAANTs (Veículos Aéreos Autônomos Não Tripulados) estão cada vez mais presentes na engenharia, como, por exemplo, na aquisição de imagens aéreas e monitoramento ISCOLD (2010), inspeção de dutos e linhas de transmissão de energia HRABAR (2010), mapeamento e pulverização de plantações GRZONKA (2012), visando redução de custo, aumento de segurança e a preservação da vida humana.

No desenvolvimento dos veículos aéreos, existem diversas linhas de pesquisa, dentre elas o desenvolvimento de robustos algoritmos de controle de baixo nível, tornando possível planejar seu movimento, detectar e evitar obstáculos e o voo autônomo, com capacidade de se localizar no ambiente. O quadrotor possui uma topologia e dinâmica diferenciada, o que permite manobras mais precisas em comparação a outros veículos aéreos. Um empecilho é o desafio no controle para integrar sensores, atuadores e inteligência do sistema, que deve ser leve e com tempo de operação muito rápido. Sistemas dinâmicos não-lineares, como o quadrotor, são desafiadores devido ao fato de seu comportamento não ser previsível e periódico. A análise de sistemas deste tipo deve ser feita cautelosamente, desde sua modelagem matemática até a execução final do projeto para o sistema.

A modelagem dos sistemas dinâmicos é uma das etapas mais importantes para a realização de projetos. Para o modelo obtido ser uma representação fiel ao sistema, os parâmetros do modelo devem ser estimados adequadamente. Experimentos de estimação paramétrica ou identificação de sistemas podem ser executados para se obter um modelo mais fiel. Contudo, a qualidade do sinal de excitação e do método de estimação dos parâmetros são críticos para o sucesso do experimento. Em COSTA (2017), uma nova metodologia , denominada SOESGOPE - (Sub-Optimal Excitation Signal Generation and Optimal Parameter Estimation) - foi apresentada. Seu intuito é obter a estimação dos parâmetros de sistemas não-lineares, e neste trabalho ela é utilizada e avaliada para a obtenção do modelo do quadrotor mais confiável.

Tal método é responsável pela obtenção de um sinal de excitação confiável (OID - Optimal Input Design) e posteriormente pela estimação dos parâmetros do modelo (OPE - Optimal Parameter Estimation), apresentando bons resultados para estimação de parâmetros de robôs móveis COSTA (2018). Neste trabalho, o método será avaliado através de simulações de um quadrotor, de forma a verificar a viabilidade do mesmo para aplicações com VAANTs.

Este trabalho é dividido como se segue. Na Seção 2 está representada a metodologia de estimação de parâmetros utilizada no trabalho. Na Seção 3 é apresentado o modelo dinâmico de um quadrotor e a técnica de controle aplicada para o veículo aéreo. Na Seçõa 4 são apresentadas definições para a utilização da metodologia nos experimentos realizados.Na Seção 5 são discutidos os resultados obtidos em simulações. Por fim, a Seção 6 corresponde as conclusões do trabalho. ^ Agradecemos a FAPEMIG, CAPES, CNPq e UFJF pelo suporte
a este trabalho.

\section{ESTIMAÇÃO DE PARÂMETROS PARA IDENTIFICAÇÃO DE SISTEMAS}

A estimação de parâmetros é uma etapa do processo de identificação de sistemas AGUIRRE (2004). Existem diversos métodos de estimação ótima de parâmetros (OPE). Para tal, é necessário um sinal de excitação rico, que excite suficientemente o sistema de forma que os parâmetros estimados se apliquem a uma série de outras entradas, e não apenas ao sinal específico da excitação inicialmente utilizada. A escolha do sinal é fundamental, devido ao fato de exercer influência, não apenas no resultado final, mas na dinâmica e duração do experimento. Adicionalmente, deve-se levar em consideração ISERMANN (2005):

- a geração do sinal deve ser trivial com descrição matemática simples;

- deve ser aplicável ao processo;

- deve prover excitação adequada a dinâmica do sistema em estudo.

\subsection{A metodologia SOESGOPE}

A qualidade do sinal de excitação é crítica para o processo de estimação paramétrica, e sinais com características de ruído branco tendem a ser persistentemente excitantes AMSTRöM (2013). Contudo, outras qualidades são desejáveis ao sinal de excitação: que sua duração não seja muito longa, que não consuma muitos recursos, que não seja destrutiva ou perigosa para o sistema, etc.

A metodologia SOESGOPE é composta por dois eficientes algoritmos de otimização dispostos em uma estratégia de camada dupla. A camada interna é responsável por encontrar a estimativa ótima do conjunto de parâmetros " $a$ posteriori" $\hat{\boldsymbol{\Gamma}}^{+}$para um dado sinal de excitação, através da minimização do erro quadrático dos sinais de saída da planta e do obtido pelo modelo paramétrico $\mathcal{M}(\Gamma)$ através da minimização:

$$
f_{o}(\cdot)=\left\{\begin{array}{l}
\underset{\hat{\boldsymbol{\delta}}^{+}}{\operatorname{minimize}} \sum_{k=0}^{t_{u}} \varepsilon(k) \\
\text { sujeito a }-\boldsymbol{\Delta} \leq \hat{\boldsymbol{\delta}}^{+} \leq \boldsymbol{\Delta}
\end{array}\right.
$$

no qual, $\varepsilon(k)=\left\|\hat{\boldsymbol{x}}^{+}(k)-\boldsymbol{x}(k)\right\|+\left\|\hat{\boldsymbol{y}}^{+}(k)-\boldsymbol{y}(k)\right\|$ é a diferença quadrática entre o estado do modelo estimado $\hat{\boldsymbol{x}}^{+}$e o estado do sistema $\boldsymbol{x}$, somado à diferença quadrática entre a saída do modelo estimado $\hat{\boldsymbol{y}}^{+}$e a saída do sistema $\boldsymbol{y}$, no instante $k$. O vetor $\boldsymbol{\Delta}$ determina a faixa de busca aceitável para os parâmetros.

Utiliza o algoritmo SBIP, devido ao fato de apresentar maior estabilidade numérica, tempo de convergência reduzido e possibilidade de usar as variáveis duais (variáveis de folga - slack variables - e os multiplicadores de Lagrange) para analisar as soluções nas fronteiras de otimização OLIVEIRA (2015).

O resultado desta camada é a variação de parâmetros ótima $\hat{\boldsymbol{\delta}}^{+}$, que minimiza o erro do sinal de saída da planta com o do modelo $\mathcal{M}\left(\hat{\boldsymbol{\Gamma}}^{+}\right)$, fazendo $\hat{\boldsymbol{\Gamma}}^{+}=\hat{\boldsymbol{\Gamma}}^{-}+\hat{\boldsymbol{\delta}}^{+}$, onde $\hat{\boldsymbol{\Gamma}}^{-}$é o conjunto de parâmetros "a priori", uma estimativa anterior dos parâmetros do sistema COSTA (2018). 
A camada externa, tem por objetivo gerar e avaliar cada sinal utilizado pela camada interna, utilizando uma função custo multi-objetivo:

$$
\begin{gathered}
\underset{\boldsymbol{u}, \hat{\boldsymbol{\delta}}^{+}}{\operatorname{minimize}} \quad \kappa_{1} \Theta(\cdot)+\kappa_{2} f_{o}(\cdot)+\kappa_{3} f_{\hat{\delta}}(\cdot) \\
\text { onde } \kappa_{1} \ggg \kappa_{2}, \kappa_{3}
\end{gathered}
$$

em que $\kappa_{1}, \kappa_{2}, \kappa_{3} \in \mathbb{R}_{\geq 0}$ são ganhos de ponderação das funções objetivo, $f_{o}(\cdot)$, e $f_{\hat{\delta}}(\cdot)$ representam a avaliação do sinal em respeito à precisão de saída, e função de recuperabilidade de parâmetros, respectivamente.

A função $\Theta(\cdot)$ representa a avaliação do sinal em relação às restrições de tempo, saída e espaço de estados:

$$
\Theta(\cdot):=\left\{\begin{array}{l}
\frac{1}{\kappa_{1}} t_{u}+\frac{\kappa_{t}}{\kappa_{1}} f_{V}\left(\tilde{\boldsymbol{x}}^{p}\right)+\frac{\kappa_{t}}{\kappa_{1}} f_{V}\left(\tilde{\boldsymbol{y}}^{p}\right), \text { se } \sum_{\tilde{\boldsymbol{x}}^{p}, \tilde{\boldsymbol{y}}^{p}, t_{u}} \Psi(\cdot) \leq \alpha \\
\Psi\left(\tilde{\boldsymbol{x}}^{\boldsymbol{p}}\right)+\Psi\left(\tilde{\boldsymbol{y}}^{\boldsymbol{p}}\right)+\Psi\left(t_{u}\right)+1, \quad \text { caso contrário }
\end{array}\right.
$$

no qual, $t_{u}$ é a duração do experimento, $\kappa_{t}$ um ganho de ponderação, $f_{V}$ é o volume do Axis-Aligned Bounding Box - AABB, que contem os estados $\tilde{\boldsymbol{x}}^{\boldsymbol{p}}$ e as saídas $\tilde{\boldsymbol{y}}^{\boldsymbol{p}}$, de um modelo $\mathcal{M}\left(\tilde{\boldsymbol{\Gamma}}^{p}\right)$, com parâmetros perturbados $\tilde{\boldsymbol{\Gamma}}^{p}$. As funções $\Psi(\cdot)$ são penalidades cumulativas, avaliadas em tempo, estados e saídas, que devem estar entre limites mínimos e máximos. Os sinais gerados são parametrizados de modo a serem similares a sinais APRBS - Amplitude Mudulated Pseuro Randomic Binary Signals, e sua otimização é feita por uma variação do algoritmo Particle Swarm Optimization (PSO), proposto por HONORIO (2010). Para aumentar taxa de convergência, é utilizado $\alpha$ constrain, que permite nas primeiras iterações que algumas penalidades sejam ignoradas, através de um valor de uma variável $\alpha$, que diminui com o número de iterações do PSO.

Deste modo, é possível se ponderar a importância de cada métrica com os ganhos $\kappa_{1}, \kappa_{2}, \kappa_{3}$ e $\kappa_{t}$, de modo a obter sinais (sub)ótimos de excitação $\boldsymbol{u}^{\oplus}$, com duração adequada, que utilize uma região confinada e segura do espaço de estados, e que tenham características de excitação condizentes com as necessidades do sistema.

\section{MODELO DE UM QUADROTOR}

A definição do modelo dinâmico inicia-se com a representação matemática da planta em estudo. Para a modelagem simplificada de um quadrotor, considera-se uma esfera maciça no centro, com massa $M$, raio $R$ e massas pontuais $m$ localizadas a uma distância $l$ do centro. Na Tabela 1 , são indicados os valores de tais parâmetros considerados neste trabalho:

Tabela 1. Parâmetros estruturais do projeto.

\begin{tabular}{c|c}
\hline \hline Parâmetros & Valores \\
\hline$m$ & $0,105 \mathrm{~kg}$ \\
$l$ & $0,250 \mathrm{~m}$ \\
$M$ & $1,00 \mathrm{~kg}$ \\
$R$ & $0,100 \mathrm{~m}$ \\
\hline \hline
\end{tabular}

Além dos parâmetros mensuráveis coletados, existem dois parâmetros que podem ser obtidos experimentalmente, que são as constantes que relacionam de modo linear a força dos atuadores com o sinal PWM (Pulse Width Modulation) aplicado aos motores, $k_{1}$ e $k_{2}$. Tais constantes serão estimadas utilizando a metodologia SOESGOPE dada uma estimativa inicial.

O modelo dinâmico do quadrotor em questão é definido através da representação matemática do modelo para o controle angular do veículo, em um primeiro momento desprezando $x, y$ e $z$ da aeronave:

$$
\begin{gathered}
\left(\begin{array}{c}
\dot{\phi} \\
\dot{\theta} \\
\dot{\psi}
\end{array}\right)=\left(\begin{array}{ccc}
1 & \sin \phi \tan \theta & \cos \phi \tan \theta \\
0 & \cos \phi & -\sin \phi \\
0 & \frac{\sin \phi}{\cos \theta} & \frac{\cos \phi}{\cos \theta}
\end{array}\right)\left(\begin{array}{l}
p \\
q \\
r
\end{array}\right) \\
\left(\begin{array}{c}
\dot{p} \\
\dot{q} \\
\dot{r}
\end{array}\right)=\left(\begin{array}{l}
\frac{J_{y}-J_{z}}{J_{x}} q r \\
\frac{J_{z}-J_{x}}{J_{y}} p r \\
\frac{J_{x}-J_{y}}{J_{z}} p q
\end{array}\right)+\left(\begin{array}{c}
\frac{1}{J_{x}} \tau_{\phi} \\
\frac{1}{J_{y}} \tau_{\theta} \\
\frac{1}{J_{z}} \tau_{\psi}
\end{array}\right)
\end{gathered}
$$

Os momentos de inércia em cada eixo, podem ser calculados da seguinte forma:

$$
\begin{aligned}
& J_{x}=\frac{2 M R^{2}}{5}+2 l^{2} m \\
& J_{y}=\frac{2 M R^{2}}{5}+2 l^{2} m \\
& J_{z}=\frac{2 M R^{2}}{5}+4 l^{2} m
\end{aligned}
$$

Dado o modelo dinâmico, é possível realizar o controle angular utilizando diversas técnicas de controle distintas.

A escolha do tipo de controlador a ser utilizado deve ser decidida com base na natureza da planta e nas condições de operação, incluindo certas considerações como segurança, custo, disponibilidade, confiabilidade, precisão, peso e tamanho OGATA (1996). A técnica de controle PID é a combinação das ações de controle proporcional, integral e derivativo. Esta ação combinada tem as vantagens individuais de cada uma das três ações de controle OGATA (1996). A Equação de um controlador PID é dada por:

$$
u(t)=K_{p} e(t)+\frac{K_{p}}{T_{i}} \int_{0}^{t} e(t) d t+K_{p} T_{d} \frac{\mathrm{d} e(t)}{\mathrm{d} t}
$$

Existem diversas técnicas para se projetar o controlador PID através do ajuste de seus parâmetros. Neste trabalho, o método utilizado foi o Twiddle.

O método Twiddle possui grande versatilidade, podendo ser aplicado aos mais diversos processos, tendo sido aplicado inclusive em quadrotores GOLDSTEIN (2012). O método foi criado baseado em estratégia de sintonia fina e nos critérios de desempenho de integrais de erro, para gerar uma técnica que minimize o erro médio medido na resposta da planta. Para executar o método, é importante conseguir reproduzir a performance da planta a ser controlada em uma simulação, pois para atingir a minimização do erro podem ser necessárias muitas iterações.

O método tem como parâmetros um vetor $P$ de ganhos a serem inseridos no controlador $\operatorname{PID}\left(P=\left[K_{p}, T_{i}, T_{d}\right]\right)$, 
um vetor $d P$ de variação diferencial para os ganhos de $P$, e uma porcentagem de variação $\xi$ para os parâmetros do vetor $d P$. Inicialmente, os valores de $P$ são inseridos no controlador PID, e a simulação executa um teste com estes valores, medindo o erro total $E$ obtido. A seguir, o primeiro parâmetro de $P\left(K_{p}\right)$ é acrescido de $d P$ e o teste é refeito. Caso o valor de $E$ seja menor do que na iteração anterior, o que indica que a variação feita foi na direção certa, o valor de $K_{p}$ continua sendo acrescido de $d P$. Caso contrário, tenta-se variar $K_{p}$ na direção contrária, decrescendo este por $d P$. Obtendo piora de resultado em ambas as direções, tenta-se novamente reduzindo o valor de $d P$ por $\xi$, e assim sucessivamente até que a variação de resultado seja desprezível, passando então para o próximo parâmetro $\left(T_{i}\right)$. Após executar o processo por todos os parâmetros, são obtidos os valores de $K_{p}, T_{i}$ e $T_{d}$ para os quais são obtidos os menores valores de erro atingidos. O método não garante atingir precisamente o mínimo global do sistema, porém se mostra altamente eficiente para a grande maioria dos casos NORVIG (2019).

Foram realizadas duas abordagens para o algoritmo Twiddle, uma para determinar o controlador para os eixos roll e pitch, que são simétricos, e uma para determinar o controlador para o eixo yaw. Para a primeira abordagem, o algoritmo realizou 145 iterações e para a segunda, 132 iterações, procurando obter o menor valor para a integral do erro absoluto do sistema. Os ganhos obtidos para os controladores são apresentados na Tabela 2 :

Tabela 2. Ganhos dos Controladores de $\phi, \theta$ e $\psi$ - Método Twiddle

\begin{tabular}{c|c|c|c}
\hline \hline & $K_{p}$ & $T_{i}$ & $T_{d}$ \\
\hline$\phi, \theta$ & 0.0018 & 0.0007 & 0.0072 \\
$\psi$ & 0.0028 & 0.0014 & 0.0057 \\
\hline \hline
\end{tabular}

\section{PARAMETRIZAÇÃO DO EXPERIMENTO}

Nesta seção serão apresentadas algumas definições importantes para a utilização da metodologia SOESGOPE, que serão utilizadas nos experimentos. O quadrotor simulado será considerado como um sistema $\mathcal{M}(\boldsymbol{\Gamma})$, cujos parâmetros $\boldsymbol{\Gamma}=\left[k_{1}, k_{2}\right]^{T}$ serão estimados, com os demais mantidos constantes. A região de busca dos parâmetros foi definida $\boldsymbol{\Delta}=[0.03,0.05]^{T}$, de modo que os parâmetros encontrados estarão confinados a $\hat{\boldsymbol{\Gamma}}^{-} \pm \boldsymbol{\Delta}$.

O sistema é composto por $n=6$ estados $\boldsymbol{x}=[p, q, r, \phi, \theta, \psi]^{T}$, em que $p, q$ e $r$ são as velocidades angulares em cada eixo, roll, pitch e yaw, respectivamente. Serão consideradas $m=3$ saídas controláveis, os ângulos da aeronave $\boldsymbol{y}=[\phi, \theta, \psi]^{T}$.

O modelo ainda considera que os estados e as saídas estão corrompidos por ruído gaussiano branco $\mathcal{N}(0, \sigma)$, com intuito de emular os ruídos dos processos reais. O desvio padrão do ruído presente nos estados e nas saídas foram considerados $\boldsymbol{\sigma}_{\boldsymbol{x}}=\left[10^{-3}, 10^{-3}, 10^{-3}, \frac{\pi}{180}, \frac{\pi}{180}, \frac{\pi}{180}\right]^{T}$ e $\boldsymbol{\sigma}_{\boldsymbol{y}}=\left[\frac{\pi}{180}, \frac{\pi}{180}, \frac{\pi}{180}\right]^{T}$, respectivamente.

Para preservar o quadrotor, foi delimitada uma região de segurança de operação no espaço de estados (e portanto também nas saídas). Os máximos e mínimos permitidos para os estados nesse experimentos foram $\mathcal{O} \boldsymbol{x}_{\max }=$ $\left[10^{5}, 10^{5}, 10^{5}, \frac{2 \pi}{3}, \frac{2 \pi}{3}, \frac{2 \pi}{3}\right]^{T}$ e $\mathcal{O} \boldsymbol{x}_{\min }=\left[-10^{5},-10^{5},-10^{5}\right.$, $\left.-\frac{2 \pi}{3},-\frac{2 \pi}{3},-\frac{2 \pi}{3}\right]^{T}$, respectivamente. Note que apenas as saídas (os três últimos estados) estão de fato limitadas, por restrições de segurança no voo da aeronave, os estados relativos às velocidades receberam valores arbitrariamente altos, visto que não apresentam risco direto ao veículo.

Por fim, o sinal de excitação $\boldsymbol{u}^{\oplus}$ foi parametrizado de modo a gerar referências para os controladores de $\phi, \theta$ e $\psi$. Os sinais serão compostos de 5 estágios, com duração variável entre $\boldsymbol{t}_{\min }=[0.071,0.071,0.105]^{T}$ e $\boldsymbol{t}_{\max }=$ $[3.565,3.565,5.250]^{T}$. Essa duração foi escolhida de modo que o estágio mínimo tenha duração de um décimo da constante de tempo estimada para o sistema, e máximo tem a duração de cinco constantes de tempo. A amplitude das referências foi proposta de modo a respeitar as restrições angulares, de modo que não excedam $\pm \frac{5 \pi}{36}\left(\mathrm{ou} \pm 25^{\circ}\right)$.

\section{RESULTADOS E DISCUSSÕES}

Três cenários de análises foram propostos para atestar a aplicabilidade da metodologia SOESGOPE em um quadrotor. Como não é possível determinar com precisão os valores dos parâmetros de um sistema real, em cada cenário uma condição simulada será considerada a situação real dos parâmetros. Isso permitirá quantificar a eficiência da metodologia em estimar os parâmetros do VAANT.

O primeiro cenário apresenta uma situação na qual há 4 modelos "a priori" $\mathcal{M}\left(\hat{\boldsymbol{\Gamma}}^{-}\right)$diferentes. Deseja-se atestar a capacidade de cada um dos modelos gerar um sinal de excitação (sub)ótimo capaz de estimar os parâmetros corretamente de um modelo dito real.

Uma vez que, nem sempre é possível, na prática, conhecer o parâmetro real de um sistema, devido à fatores, como as incertezas de medição, um segundo cenário propõe a geração de apenas um sinal de excitação $\boldsymbol{u}^{\oplus}$, e 4 hipóteses de sistema real, ou seja, 4 conjuntos $\boldsymbol{\Gamma}$. Deseja-se atestar a capacidade do sinal em excitar estes modelos distintos, permitindo a sua correta estimação em cada cenário.

Um terceiro cenário propõe uma análise de sensibilidade dos ganhos de ponderação da otimização do sinal, para analisar seu impacto no sinal de excitação produzido.

\subsection{Cenário 1: Diferentes Modelos Iniciais}

Para o primeiro teste realizado, dado um certo conjunto de parâmetros considerados como reais, analisa-se a geração de sinais sub(ótimos) a partir de estimativas iniciais diferentes para verificação e validação dos sinais gerados.

O modelo do veículo aéreo considerado como "real" para os testes é definido pelo conjunto de parâmetros indicado na Tabela 1 , considerando as constantes a serem estimadas conforme a Tabela 3 .

Tabela 3. Cenário 1: Parâmetros do quadrotor "real" $-\boldsymbol{\Gamma}$.

\begin{tabular}{c|c|c}
\hline \hline Parâmetro & Valor & Unidade \\
\hline$k_{1}$ & 0.0268 & s.u \\
$k_{2}$ & 0.0147 & s.u \\
\hline \hline
\end{tabular}


Os parâmetros $k_{1}$ e $k_{2}$ são os parâmetros desconhecidos do modelo, e a tabela indica valores aproximados das constantes. Tais constantes, que transformam de forma linear o comando PWM em força, são obtidas aproximadamente de forma experimental.

Alguns cenários de parâmetros $k_{1}$ e $k_{2}$ iniciais foram gerados para se obter os sinais sub(ótimos) referente a cada um dos casos, conforme indicado na Tabela 4. O primeiro caso se refere ao modelo "real", no segundo caso há um acréscimo de $25 \%$ aos valores do modelo real, no terceiro caso há um acréscimo de $50 \%$, e no quarto caso há um decréscimo de $40 \%$. Os ganhos de ponderação utilizados no primeiro e segundo cenários foram $\kappa_{1}=100000, \kappa_{2}=100$, $\kappa_{3}=1000$ e $\kappa_{t}=1$.

Tabela 4. Geração do conjunto de sinais

\begin{tabular}{ccccc}
\hline \hline Caso & 1 & 2 & 3 & 4 \\
\hline$\hat{\boldsymbol{\Gamma}}^{-}-k_{1}$ & 0.0268 & 0.0335 & 0.0402 & 0.0161 \\
$\hat{\boldsymbol{\Gamma}}^{-}-k_{2}$ & 0.0147 & 0.0184 & 0.0220 & 0.0088 \\
$\tilde{\boldsymbol{\Gamma}}^{\boldsymbol{p}_{-} k_{1}}$ & 0.0278 & 0.0348 & 0.0417 & 0.0167 \\
$\tilde{\boldsymbol{\Gamma}}^{\boldsymbol{p}_{-} k_{2}}$ & 0.0189 & 0.0236 & 0.0283 & 0.0105 \\
$f_{o}(\cdot)$ & $8.720 \cdot 10^{-4}$ & $8.708 \cdot 10^{-4}$ & $8.729 \cdot 10^{-4}$ & $8.745 \cdot 10^{-4}$ \\
$f_{\hat{\delta}}(\cdot)$ & $1.446 \cdot 10^{-6}$ & $8.189 \cdot 10^{-7}$ & $6.103 \cdot 10^{-7}$ & $4.131 \cdot 10^{-8}$ \\
$f_{v}(\cdot)$ & 0.3576 & 0.3580 & 0.3172 & 0.4544 \\
\hline \hline
\end{tabular}

Para tal simulação, como pode-se analisar pela tabela, o conjunto de parâmetros do modelo inicial $\hat{\Gamma}^{-}$gerou um conjunto de parâmetros perturbados $\tilde{\Gamma}^{p}$. Para todos os casos as funções de restrições obtiveram valores pequenos, o que significa que o algoritmo obteve bons resultados.

Através dos resultados obtidos, a métrica de restrições de tempo, saída e espaço de estados retornou uma função de valor baixo em todos os casos, na faixa de $10^{-6}$. Isto significa que o algoritmo manteve a saída $y$ e os estados $x$ dentro dos limites de espaço admissíveis.

A função de precisão de saída, $f_{o}$ mede a diferença entre a saída e os estados de dois sistemas excitados pelo mesmo sinal, ou seja, o modelo inicial, $\hat{\Gamma}^{-}$, e o modelo perturbado, $\tilde{\Gamma}^{p}$, obtendo uma diferença pequena entre eles como se pode observar pela Tabela 4 , facilitando assim a estimação do conjunto de parâmetros ótimos.

A função de recuperabilidade de parâmetros, $f_{\hat{\delta}^{+}}$, avalia a persistência de excitação do sinal. É a garantia de que o conjunto de parâmetros estimados convergem para os parâmetros perturbados. É possível perceber que em todos os casos, a geração de sinal sub(ótimo) de excitação foi realizada com sucesso, garantindo assim que o conjunto de parâmetros ótimos será obtido.

Através da análise dos resultados gerados para a geração dos sinais de excitação, percebe-se que o sinais serviram para excitar de maneira suficiente o sistema e a partir daí estimar os valores ótimos dos parâmetros. A Tabela 5 possui o valor dos parâmetros estimados com os sinais gerados e o erro quadrático da estimação.

A estimação dos parâmetros foi satisfatória, visto que o conjunto de parâmetros estimados está próximo ao conjunto de parâmetros "reais"como se pode analisar através dos baixos valores de erro quadrático de estimação $\left(\Sigma_{\Gamma}\right)$ obtidos nas estimações.
Tabela 5. Estimação de parâmetros ótimos " $a$ posteriori" $-\hat{\Gamma}^{+}$.

\begin{tabular}{c|cc|c}
\hline \hline \multirow{2}{*}{ Caso } & \multicolumn{2}{|c|}{$\hat{\Gamma}^{+}$} & \multirow{2}{*}{$\Sigma_{\Gamma}$} \\
\cline { 2 - 3 } & $k_{1}$ & $k_{2}$ & \\
\hline 1 & 0.0267 & 0.0149 & $4.1307 \cdot 10^{-8}$ \\
2 & 0.0269 & 0.0148 & $1.4820 \cdot 10^{-8}$ \\
3 & 0.0269 & 0.0147 & $7.9101 \cdot 10^{-9}$ \\
4 & 0.0268 & 0.0147 & $2.2307 \cdot 10^{-9}$ \\
\hline \hline
\end{tabular}

5.2 Cenário 2: Estimação a partir do mesmo sinal de excitação

Inicialmente, a abordagem realizada previa cinco modelos iniciais e a partir deles, cinco conjuntos de sinais de excitação eram gerados para a estimação dos parâmetros, um conjunto para cada modelo distinto.

Uma verificação acerca da metodologia a ser feita, é a possibilidade de que, a partir de um modelo inicial, neste caso, considerado o modelo "real", gerar apenas um conjunto de sinais sub(ótimos) de excitação, para a partir deste conjunto de sinais, estimar diversos modelos distintos. Em suma, o mesmo conjunto de sinais gerados pelo modelo "real", com: $k_{1}=0.0268$ e $k_{2}=0.0147$, deve conseguir estimar o conjunto de parâmetros ótimos a partir de modelos iniciais diferentes.

Quatro casos de modelos iniciais foram observados. O primeiro caso se refere ao modelo com acréscimo de $10 \%$ dos valores do modelo "real", o segundo caso há um acréscimo de 30\%, o terceiro caso há um decréscimo de $30 \%$ e o quarto caso há um acréscimo de $25 \%$ no parâmetro $k_{1}$ e um decréscimo de $25 \%$ no parâmetro $k_{2}$. Os resultados de estimação obtidos estão indicados na Tabela 6 .

Tabela 6. Estimação de parâmetros ótimos " $a$ posteriori" a partir do mesmo sinal $-\hat{\Gamma}^{+}$.

\begin{tabular}{c|cc|cc|c}
\hline \hline \multirow{2}{*}{ Caso } & \multicolumn{2}{|c|}{$\hat{\Gamma}^{-}$} & \multicolumn{2}{|c|}{$\tilde{\Gamma}^{+}$} & \multirow{2}{*}{$\Sigma_{\Gamma}$} \\
\cline { 2 - 5 } & $k_{1}$ & $k_{2}$ & $k_{1}$ & $k_{2}$ & \\
\hline 1 & 0.0295 & 0.0162 & 0.0267 & 0.0148 & $1.8015 \cdot 10^{-8}$ \\
2 & 0.0348 & 0.0191 & 0.0268 & 0.0147 & $5.687 \cdot 10^{-10}$ \\
3 & 0.0188 & 0.0103 & 0.0266 & 0.0147 & $4.1228 \cdot 10^{-8}$ \\
4 & 0.0335 & 0.0011 & 0.0265 & 0.0149 & $1.1764 \cdot 10^{-7}$ \\
\hline \hline
\end{tabular}

Na Tabela 6 são indicados os valores dos conjuntos de parâmetros iniciais e os parâmetros estimados. Nota-se que a metodologia consegue estimar os parâmetros ótimos do sistema, partindo de modelos iniciais distintos excitados por um mesmo conjunto de sinais, analisando os valores dos parâmetros estimados e o erro quadrático encontrado na estimação.

\subsection{Cenário 3: Sensibilidade aos Ganhos de Ponderação}

Por fim, um último experimento realizado, foi o de sensibilidade aos cenários de ganhos de ponderação, das métricas da metodologia, diferentes.

Para cada caso, como indicado na Tabela 7 , foram obtidos os sinais de excitação e a partir deles a estimativa dos parâmetros $k_{1}$ e $k_{2}$. O modelo inicial considerado, foi o modelo "real" com: $k_{1}=0.0268$ e $k_{2}=0.0147$.

A partir dos parâmetros estimados, é perceptível que mesmo com a alteração dos ganhos de ponderação, os 
parâmetros ainda estão sendo bem estimados, logo a metodologia funciona de maneira com que os ganhos apenas alteram as penalidades em cada modelo, gerando ainda sinais sub(ótimos) para excitação da planta e a estimação de parâmetros ótimos.

Tabela 7. Cenários de ganhos de ponderação $\kappa_{1}, \kappa_{2}, \kappa_{3}, \kappa_{t}$.

\begin{tabular}{c|cccc|cc|c}
\hline \hline Caso & $\kappa_{1}$ & $\kappa_{2}$ & $\kappa_{3}$ & $\kappa_{t}$ & \multicolumn{2}{|c|}{$\hat{\Gamma}^{+}$} & \multirow{2}{*}{$\Sigma_{\Gamma}$} \\
\cline { 6 - 7 } & & & & & $k_{1}$ & $k_{2}$ & \\
\hline 1 & $10^{5}$ & $10^{1}$ & $10^{2}$ & $10^{-1}$ & 0.0267 & 0.0147 & $5.034 \cdot 10^{-9}$ \\
2 & $10^{5}$ & $10^{3}$ & $10^{2}$ & 1 & 0.0266 & 0.0149 & $7.886 \cdot 10^{-8}$ \\
3 & $10^{3}$ & $10^{1}$ & $10^{1}$ & 1 & 0.0267 & 0.0147 & $1.428 \cdot 10^{-8}$ \\
\hline \hline
\end{tabular}

\section{CONCLUSÕES}

Este artigo resume a aplicação da metodologia em um quadrotor para a estimação ótima de parâmetros. Um dos desafios presentes é a modelagem e implementação do controle do sistema, visto que existem diversas técnicas de sintonia de parâmetros para o controlador PID, porém, algumas delas não possuem bons resultados aplicadas em conjunto com a metodologia SOESGOPE. Devido a este fato, uma técnica de sintonia baseada em otimização foi aplicada para a construção do controlador. O método twiddle obteve ótimos resultados para a planta em questão, uma vez que tal método consiste em um loop de iterações em que o objetivo é a minimização do erro da planta do sistema controlado.

Com o sistema controlado, foi possível aplicar a metodologia para gerar sinais sub(ótimos) de excitação e, a partir deles, estimar os parâmetros desconhecidos do sistema em diversos cenários obtendo um desempenho satisfatório. As simulações realizadas obtiveram bons resultados para a estimação ótima dos seus parâmetros. Inicialmente, uma simulação com os parâmetros do sistema "real"gerou um conjunto de sinais de excitação e a partir deles os parâmetros foram estimados. Sem penalidades na estimação dos parâmetros, eles foram próximos aos parâmetros considerados reais, com valor do erro quadrático da estimação baixo. Em seguida, mais três conjuntos de sinais diferentes foram gerados para tentar obter a estimação dos mesmos parâmetros, e novamente o método obteve sucesso. Analisando o primeiro cenário considerado, através da estimação ótima de parâmetros e o erro quadrático encontrado, a metodologia se mostra aplicável ao gerar conjuntos de sinais sub(ótimos) capazes de excitar o sistema, mesmo considerando modelos iniciais distintos. Já o segundo cenário viabiliza a metodologia a estimar os parâmetros de um sistema, com modelos iniciais distintos, mas partindo de um mesmo conjunto de sinais sub(ótimos). Por fim, no terceiro cenário, testes para analisar a sensibilidade de ganhos de ponderação das métricas da metodologia demonstrou a capacidade do método, destacando que mesmo alterando o peso dos ganhos, os parâmetros foram bem estimados. Em todos os três cenários, o erro quadrático médio da estimação foi consideravelmente pequeno, em que, o maior se encontra na faixa de $10^{-7}$, o que indica que os parâmetros foram bem estimados.

A metodologia se mostra uma alternativa eficaz para estimar parâmetros de sistemas, sendo possível aplicála em casos distintos, como analisado nos dois primeiros cenários presentes no trabalho. Para cada aplicação é possível determinar quais métricas da metodologia são mais relevantes e, a partir daí, definir os valores dos ganhos das constantes de ponderação, uma vez que a estimação é garantida.

\section{REFERÊNCIAS}

AGUIRRE, L.A. (2004). Introdução à identificação de sistemas - Técnicas lineares e não-lineares aplicadas a sistemas reais. Editora UFMG.

AMSTRöM, K. J.; WITTENMARK, B. (2013). Adaptive control. Mineola: Dover Publications, Inc.

COSTA, E.B. (2017). Metodologia de otimização em dois níveis para a geração de sinal sub-Ótimo de excitação e estimação de parâmetros de sistemas não lineares restritos.

COSTA, E.B.e.a. (2018). Persistently-exciting signal generation for optimal parameter estimation of constrained nonlinear dynamical systems. In ISA TRANSACTIONS., 231-241.

GOLDSTEIN, R.e.a. (2012). Beohawk: Autonomous quadrotor. In Symposium on Indoor Flight Issue., 1-11.

GRZONKA, S.; GRISETTI, G.B.W. (2012). A fully autonomous indoor quadrotor. In Robotics, IEEE Transactions on, IEEE., 90-100.

HONORIO, L.e.a. (2010). Solving optimal power ow problems using a probabilistic -constrained evolutionary approach. In IET generation, transmission distribution., 674-682.

HRABAR, S.; MERZ, T.F.D. (2010). Development of an autonomous helicopter for aerial powerline inspections. In IEEE. Applied Robotics for the Power Industry (CARPI)., 1-6.

ISCOLD, P.; PEREIRA, G.e.a. (2010). Development of a hand-launched small uav for ground reconnaissance. In Aerospace and Electronic Systems, IEEE Transactions on, IEEE., 335-348.

ISERMANN, R. (2005). Mechatronic systems: fundamentals. London, UK: Springer-Virlag.

NORVIG, P.; THRUN, S. (2019). Udacity - intro to artificial intelligence. https://www.udacity.com/course/ intro-to-artificial-intelligence--cs271.

OGATA, K. (1996). Engenharia de controle moderno. Prentice Hall.

OLIVEIRA, E.J.e.a. (2015). An optimal power ow based on safety barrier interior point method. In International Journal of Electrical Power Energy Systems., 977-985. 